\title{
Estudo de prevalência de olho seco em estudantes universitários
}

\author{
Isabela Yang*, Isabella B. I. Sacho, Gabriel A. Lopes, Marina P. Vizotto, Breno D. Gregorio, Nelson O. W. \\ Choueri, Paula R. M. Costa, Monica Alves
}

\section{Resumo}

O olho seco é uma doença multifatorial, complexa e comum na superfície ocular e no filme lacrimal que resulta em desconforto e distúrbio visual ${ }^{1}$. Os índices de prevalência têm uma ampla variação e são, majoritariamente, baseados em estudos em população idosa. Por meio de dois questionários validados na língua portuguesa, esse estudo busca avaliar os fatores de risco na população mais jovem dos estudantes de medicina da UNICAMP, para compreender os sintomas, os sinais clínicos e os fatores de risco mais associados ${ }^{2},{ }^{3}$.

\section{Palavras-chave:}

Olho seco, epidemiologia, jovens

\section{Introdução}

Estudo clínico observacional, transversal que incluiu 348 voluntários alunos de medicina da Universidade de Campinas (Unicamp). Todos esses responderam os questionários auto-aplicáveis sobre sintomas de Olho Seco: Ocular Surface Disease Index (OSDI) e um questionário utilizado no Women Health Study (WHS), traduzidos e validados no português. Foram abordados fatores de risco como: uso de lente de contato, horas de uso do aparelho eletrônico, medicações, cirurgia ocular e doença sistêmica associada com o olho seco. Aqueles que preencheram critérios de olho seco foram encaminhados para o Departamento de Oftalmologia da Unicamp para análise clínica e exame físico que investigam sinais de olho seco, incluindo a avaliação objetiva não invasiva da superfície ocular (Keratograph $5 \mathrm{M})$, superfície ocular com coloração de fluoresceína e com lisamina verde e Teste de Schirmer.

\section{Resultados e Discussão}

A idade média dos participantes é de 22.8土4.2 anos de idade, sendo $53.2 \%$ mulheres e $46.8 \%$ homens. $\mathrm{O}$ valor do questionário OSDI foi $14.7 \pm 14.5,29,3 \%$ dos participantes obtiveram pontuação acima de 22 pontos, valor de corte, enquanto que para o WHS, é de $17,3 \%$. Os fatores de riscos mais frequentes foram uso de aparelhos eletrônicos em $68,7 \%$ dos casos, menos de seis horas de sono em $40,6 \%$, uso de lentes de contato em $25,7 \%$, uso de contraceptivo oral em $23,6 \%$ e antidepressivos em 11,7\%. As maiores pontuações OSDI foram no uso da Isotretinoína, cirurgia ocular e lente de contato, sendo maiores ainda no sexo feminino. Avaliação também demonstrou leve sinais de disfunção na superfície ocular, disfunção das glândulas de meibômio e instabilidade do filme lacrimal.

Figura 1. Sintomas de Olho Seco nos alunos de medicina

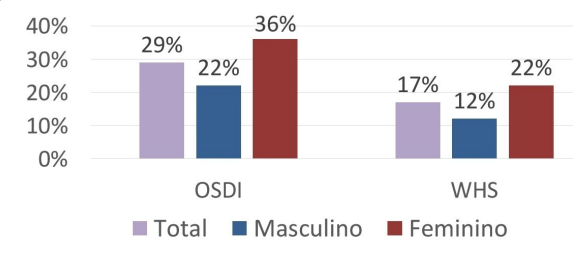

\section{Conclusões}

A prevalência de olho seco foi de $29,3 \%$ de acordo com o OSDI e de $17,3 \%$ de acordo com o questionário WHS. Essas prevalências são mais altas do que aquela encontrada no estudo anterior na população brasileira acima de 40 anos, que apresenta $12,8 \%$ de olho seco (WHS). A análise clínica dos estudantes também demonstrou sinais de leve disfunção da superfície ocular, alteração na glândula meibomiana e instabilidade no filme lacrimal. Essa amostra populacional jovem aparenta alta taxa de prevalência de olho seco, enfatizando a futura investigação nessa faixa etária para melhor compreensão, abordagem e tratamento clínico.

\section{Agradecimentos}

Agradeço aos meus familiares, amigos, professores, instituições de ensino, que contribuíram para o crescimento acadêmico e para as oportunidades em congressos nacionais e internacionais.

Instituição de fomento: PIBIC CnPQ 2018/2019

Fapesp: 2014/ 19138-5

\section{Referência}

1 Craig, J. P. et al. TFOS DEWS II Definition and Classification Report. Ocul Surf 15, 276-283, doi:10.1016/j.jtos.2017.05.008 (2017).

2 Stapleton, F. et al. TFOS DEWS II Epidemiology Report. Ocul Surf 15, 334-365, doi:10.1016/j.jtos.2017.05.003 (2017).

3. Sullivan, D. A. et al. Report of the TFOS/ARVO Symposium on global treatments for dry eye disease: an unmet need. Ocul Surf 10, 108-116, doi:10.1016/j.jtos.2012.02.001 (2012).

4. Santo, R. M., Ribeiro-Ferreira, F., Alves, M. R., Epstein, J. \& Novaes, P. Enhancing the cross-cultural adaptation and validation process: linguistic and psychometric testing of the Brazilian-Portuguese version of a self-report measure for dry eye. J Clin Epidemiol 68, 370-378, doi:10.1016/j.jclinepi.2014.07.009 (2015).

5. Schaumberg, D. A. et al. Development and validation of a short global dry eye symptom index. Ocul Surf 5, 50-57 (2007).

6 Gulati, A. et al. Validation and repeatability of a short questionnaire for dry eye syndrome. Am J Ophthalmol 142, 125-131, doi:10.1016/j.ajo.2006.02.038 (2006).

7 Wolffsohn, J. S. et al. TFOS DEWS II Diagnostic Methodology repoda versão em português de um questionário para sintomas de olho seco. Arq. Bras. Oftalmol. [online]. 2017, vol.80, n.1, pp.14-16. ISSN 0004-2749. http://dx.doi.org/10.5935/0004-2749.20170005. rt. Ocul Surf 15, 539-574, doi:10.1016/j.jtos.2017.05.001 (2017).

CASTRO, Julia Silvestre de et al. Tradução e validação 